\title{
COMPARAÇÃO DOS EFEITOS DA IMERSÃO EM ÁGUA FRIA COM APLICAÇÃO DE PLACEBO NA VARIÁVEL CREATINA QUINASE
}

Gabriela de Carvalho ${ }^{1}$, Jaqueline Santos Silva ${ }^{1,2}$, Franciele Marques Vanderlei ${ }^{1,3}$, Jéssica Kirsch Michelleti, Aryane Flauzino Machado ${ }^{1,4}$, Aline de Castilho Almeida ${ }^{1,4}$, Italo Ribeiro Lemes ${ }^{1,4}$, Malu dos Santos Siqueira ${ }^{1,2}$, Larissa Rodrigues Souto ${ }^{1,2}$, Jayme Netto Junior ${ }^{1,3}$, Carlos Marcelo Pastre ${ }^{1,3}$

"Universidade Estadual Paulista "Júlio de Mesquita Filho" - UNESP, Faculdade de Ciências e Tecnologia, Curso de Fisioterapia, ${ }^{1,2}$ Mestrado em Fisioterapia, ${ }^{1,3}$ Professor Doutor, Departamento de Fisioterapia, Laboratório de Fisioterapia Desportiva-LAFIDE, ${ }^{1,4}$ Doutorado em Fisioterapia. E-mail: pastre@fct.unesp.br

\section{RESUMO}

Introdução: A Imersão em água fria (IAF) ganha destaque no cenário da recuperação pós-esforço. Nesse contexto, o efeito placebo deve ser estudado para melhor entendimento da técnica. Objetivos: Analisar e comparar os efeitos da IAF quando aplicada de forma sistemática, com a condição placebo na variável creatina quinase (CK). Métodos: A amostra foi de 10 atletas de alto rendimento do sexo masculino, alocados de forma randomizada: grupo placebo (GP) e grupo intervenção recuperativa (GE). Quatro vezes na semana ao final de cada treino físico os participantes do GP foram imersos em água termoneutra por 15 minutos a $27^{\circ} \mathrm{C}$ e o GE foram submetidos a IAF por 15 minutos a 13ㅇ․ A CK foi coletada no momento basal e após duas semanas de intervenção. Todos os testes estatísticos realizados foram considerando o nível de significância de 5\%. Conclusão: A IAF quando comparada ao placebo, não reduziu níveis de CK sanguíneo.

Palavras Chave: dor, crioterapia, imersão, exercício, atletas, desempenho atlético.

\section{COMPARISON OF THE EFFECTS OF COLD WATER IMMERSION vs PLACEBO EFFECT IN CREATINE KINASE VARIABLE}

\begin{abstract}
Background: Recovery methods are commonly used in the sports. Cold Water Immersion (CWI) is highlighted in this scenario, but the placebo effect must be studied for better understanding of real technical efficiency. Objectives: Analyze and compare the effects of CWI when applied systematic way, with a placebo in creatine kinase (CK). Methods: 10 athletes high-male income, randomly allocated: placebo group (PG), intervention group (EG). Four times a week at the end of each physical training were PG participants immerse in thermoneutral water for 15 minutes at a temperature of $27^{\circ} \mathrm{C}$, Participants of EG were immerse for 15 minutes one a temperature of 13ㅇ. The Creatine Kinase was collected at baseline and after two weeks of intervention. All the testicles statistical were done considering the level of significance of $5 \%$. Conclusion: Cold water immersion compared to placebo, is not an effective recovery technique in lowering blood levels of creatine kinase.
\end{abstract}

Key Words: pain, cryotherapy, immersion, exercise, athletes, athletic performance. 


\section{INTRODUÇÃO}

O exercício físico de alta intensidade é responsável por alterações em diversos sistemas $\operatorname{orgânicos}^{(1,2)}$. Nesse cenário, técnicas recuperativas têm sido amplamente utilizadas proporcionando a restauração dos sistemas do corpo a sua condição basal, e assim, melhorando a performance $e^{(3,4)}$.

A imersão em água fria (IAF), modalidade da crioterapia, é uma das técnicas comumente descritas $^{(2,5)}$. Esta é definida como a imersão de qualquer parte corpórea em água de temperatura menor que $15^{\circ} \mathrm{C}^{(6)}$, sendo capaz de reduzir a permeabilidade dos vasos devido a vasoconstrição, temperatura corporal, com consequente diminuição do metabolismo celular e do edema muscular, além de reduzir a velocidade da condução nervosa, a atividade do fuso muscular e a dor $^{(7)}$. Alguns estudos demonstram resultados positivos em relação à IAF em aspectos clínicos, metabólicos e funcionais ${ }^{(6,7,8,9,10)}$.

Nesse cenário, a concentração sanguínea de creatina quinase (CK) é usada para verificação de lesão muscular decorrentes de atividades físicas ${ }^{(11,12,13)}$. Quanto maior a intensidade do exercício, maior será a quantidade de microtraumas musculares, desencadeando o extravasamento desta enzima para o meio extracelular ${ }^{(14)}$. O processo de aumento de CK no sangue pode estar associada à dor e desconforto músculo-tendíneo ${ }^{(15,4)}$.

Ascensão et $a l^{(10)}$ em seu estudo utilizou a IAF, durante10 minutos a $10^{\circ} \mathrm{C}$, como método de recuperação após uma partida de futebol em atletas do sexo masculino. Após a intervenção realizada, pode-se observar uma diminuição da percepção de dor muscular e redução de níveis de CK sanguíneo. Foi observado um aumento do tempo no desaparecimento de CK sanguínea no estudo de Skurvydas et $a l^{(16)}$, quando sua amostra foi submetida a dois turnos de imersão em água fria por 15 minutos cada turno, com um intervalo de 10 minutos entre eles, a uma temperatura média de $15^{\circ} \mathrm{C}$.

Além das variáveis clínicas alguns autores ${ }^{(17,18)}$ têm apontado que há uma contribuição do mecanismo psicológico para a melhora da performance dos atletas quando estes acreditam que têm recebido um tratamento benéfico. McClung e Collins ${ }^{(19)}$ em seu estudo, administraram medicamentos para melhora da performance em atletas, sendo o estudo subdividido em dois grupos, um grupo ingerindo o produto real, e o outro grupo o produto placebo. Como resultado os dois grupos obtiveram os mesmos benefícios.

Portanto, tendo em vista que a IAF é uma estratégia de recuperação pós-exercício amplamente utilizada na prática esportiva para obtenção de níveis ótimos de recuperação e, considerando que estudos realizados em campo devem ser incentivados para responder às questões referentes à eficiência da técnica em diversos sistemas orgânicos ${ }^{(9)}$ considerando o possível efeito placebo envolvido, entende-se como relevante o presente estudo. Assim, o objetivo do presente estudo foi analisar e comparar os efeitos da IAF quando aplicada de forma sistemática, com a condição placebo na concentração sanguínea de Creatina Quinase.

\section{MÉTODOS}

Trata-se de um ensaio clínico randomizado cruzado. A casuística do estudo foi composta por 10 atletas de alto rendimento do sexo masculino praticantes da modalidade de atletismo com idade entre 18 e 30 anos. 0 número de voluntários selecionados garante um poder de teste acima de $80 \%$ com nível de significância de $5 \%$.

Não foram incluídos no estudo voluntários que apresentaram pelo menos uma das seguintes características: tabagistas, uso de medicamentos que influenciam a atividade autonômica do coração, etilistas, uso de medicamentos anti-inflamatórios de forma crônica e portadores de distúrbios metabólicos e/ou endócrinos conhecidos.

Os voluntários foram devidamente informados sobre os procedimentos e objetivos deste estudo, e após concordarem, assinaram um termo de consentimento livre e esclarecido passando 
a fazer parte efetivamente do mesmo. Todos os procedimentos utilizados neste trabalho foram submetidos ao Comitê de Ética em Pesquisa da Universidade Estadual Paulista - FCT/UNESP por meio da Plataforma Brasil. Número do protocolo: 44591115.0.0000.5402

\section{INTERVENÇÕES DE RECUPERAÇÃO}

Os participantes foram alocados de forma randomizada em dois grupos de estudo: Grupo placebo (GP; $n=10)$, submetidos a uma intervenção recuperativa por 15 minutos a uma temperatura de $27 \stackrel{\circ}{ } \mathrm{C} \pm 1$ 으 $\mathrm{C}$ com adição à água uma solução de limpeza de pele com $\mathrm{pH}$ balanceado (Cetaphil, Gentle Skin Cleanser, Australia). Os participantes foram levados a acreditar na eficácia dessa loção através de uma palestra ministrada no ínicio do período de coletas. $O$ grupo experimental $(G E, n=10)$ foi submetido a IAF por 15 minutos a $13^{\circ} \mathrm{C} \pm 1 \stackrel{\circ}{\circ} \mathrm{C}$.

\section{DELINEAMENTO DO ESTUDO}

Para realização deste estudo os participantes foram submetidos a um ensaio composto por duas etapas, sendo elas: 1a Etapa: Determinação da condição basal do participante, por meio de informações pessoais (idade, tempo de treinamento e melhor resultado obtido nas competições) e avaliação dos parâmetros antropométricos (peso e estatura) ${ }^{(21)}$. Em seguida, os participantes foram submetidos à coleta de Creatina Quinase. 2a etapa: Foi iniciado o período de treinamento caracterizado por treinos periodizados estruturais, metabólicos e recuperativos composto por atividades de aquecimento, coordenação, agilidade, força muscular e potência com uma frequência de seis vezes por semana por seis semanas. Durante esse período, quatro vezes durante a semana, ao final do treino físico os participantes foram submetidos à intervenção randomizada inicialmente sendo duas semanas na IAF e duas semanas na condição placebo. A coleta de Creatina Quinase foi realizada após as duas semanas de treinamento de cada protocolo.

Após esse período, os voluntários foram trocados em relação à intervenção realizada, caracterizando assim um design cross-over (3a Etapa), ou seja, os voluntários que nas duas primeiras semanas realizaram a intervenção por IAF, em seguida (3a e 4⿳亠丷a semanas) realizaram a intervenção placebo, dependendo da randomização previamente realizada.

\section{TREINAMENTOS}

Durante esse período, o treinamento foi realizado seis vezes na semana por seis semanas, lembrando que durante quatro vezes na semana foi realizada a intervenção, dividida em três blocos, anteriormente randomizada: duas semanas de intervenção por IAF e; duas semanas de intervenção por placebo. De modo geral foram realizados treinos de musculação para membros superiores, membros inferiores e tronco, treino composto por atividades de coordenação, agilidade e potência muscular/tiros, sendo trabalhadas as capacidades aeróbicas e anaeróbicas dos voluntários.

\section{PROCEDIMENTOS}

Parâmetros antropométricos

Para determinação do Índice de Massa Corpórea (IMC) foi realizada a mensuração da estatura em posição ortostática, por meio de um estadiômetro (Sanny, American Medical do Brasil, São Paulo, Brasil) e da massa corporal por meio de uma balança digital (Tanita BC554, Iron Man/Inner Scaner). O posterior cálculo do IMC foi realizado seguindo a fórmula: IMC = peso [kg] / estatura $^{2}[\mathrm{~m}]{ }^{(20)}$.

\section{Creatina Quinase}

A creatina quinase (CK) é utilizada como marcador indireto de dano muscular ${ }^{(21)}$. Assim, para determinação da concentração enzimática de CK no plasma, serão coletados $32 \mu \mathrm{L}$ de sangue capilar da polpa digital dos sujeitos ${ }^{(22)}$. Essa punção ocorrerá por meio de uma lanceta com 
disparador automático, após a limpeza do local com álcool etílico a 95\% e secagem com algodão. A amostra sanguínea será drenada para um tubo capilar heparinizado e então pipetada para uma tira reativa de CK para sua análise no Reflotron Plus System (da marca Roche Diagnostics). O objetivo dessa coleta será verificar o dano muscular que o treinamento pode ter causado no voluntário.

\section{ANÁLISE ESTATÍSTICA}

Para análise dos dados do perfil da população foi utilizado o método estatístico descritivo e os resultados foram apresentados com valores de médias, desvios padrão, mediana e intervalo de confiança. A normalidade dos dados foi avaliada por meio do teste de Shapiro-Wills. Para comparação dentro dos grupos foi utilizado teste $t$ pareado para dados normais e teste de Wilcoxon para dados não normais e para a comparação entre os grupos foi utilizado teste $t$ não pareado para dados normais e Mann-Whitney para dados não normais. O nível de significância foi estabelecido em $\mathrm{p} \leq 0,05$ para todos os testes.

\section{RESULTADOS}

Não houve diferença estatisticamente significante entre os grupos e entre os momentos analisados (Tabela 1).

Tabela 01.Média, desvio padrão, mediana e intervalo de confiança de creatina quinase antes e após a aplicação sistemática de IAF e placebo.

\begin{tabular}{|c|c|c|c|}
\hline Índices & Momentos & IAF & Placebo \\
\hline \multirow{2}{*}{ Creatina Quinase } & Basal & $\begin{array}{c}257,91 \pm 181,32 \\
(224,00) \\
{[106,30-409,53]}\end{array}$ & $\begin{array}{c}477,25 \pm 307,79 \\
(438,00) \\
{[219,89-734,61]}\end{array}$ \\
\hline & Final & $\begin{array}{c}494,58 \pm 313,04 \\
(444,00) \\
{[232,84-756,34]}\end{array}$ & $\begin{array}{c}322,20 \pm 198,69 \\
(255,00) \\
{[138,43-505,97]}\end{array}$ \\
\hline
\end{tabular}

\section{DISCUSSÃO}

Os resultados apresentados neste estudo demonstra que não há diferença entre a IAF e o efeito placebo para a antecipação da redução da concentração sanguínea de Creatina Quinase (CK). Tal resultado abre lacunas para o questionamento sobre a real eficácia da IAF no que se refere ao aspecto fisiológico.

No cenário atual da literatura os resultados se mostram divergentes para a redução de CK sanguíneo a partir da utilização de IAF após atividades físicas de alto nível. No estudo de Jakeman et $a l^{(23)}$ foi realizado um protocolo de saltos verticais, e posteriormente os participantes foram submetidos à IAF (10 minutos, $\left.10^{\circ} \mathrm{C}\right)$, e obtiveram resultados referentes a ausência de redução dos níveis de CK no sangue. Por outro lado, outros estudos ${ }^{(10,16)}$ obtiveram resultados favoráveis sobre o uso da IAF para redução desse parâmetro. Deve-se ressaltar que foram utilizados diferentes tempos, temperaturas e protocolos de atividades físicas nos estudos apresentados, sendo esse um potente fator interferencial nos diferentes resultados.

Todos os participantes desse estudo já utilizaram em algum momento de seu treinamento a imersão em água fria para uma melhor recuperação de seus sistemas corpóreos, portanto podese dizer que a familiarização com essa técnica possa ter influenciado diretamente nos resultados dessa pesquisa. Outro ponto a ser considerado é a eficácia da maneira de levar os participantes a acreditar na nova proposta de recuperação através da adição do óleo corporal à água 
termoneutra. Dentre as limitações encontradas, deve-se ressaltar a deficiência de estudos que utilizaram a IAF em comparação ao Placebo para variável creatina quinase, sendo um fator dificultante para comparação dos resultados apresentados.

Portanto, sugere-se uma maior investigação do efeito fisiológico da IAF, quando comparada à uma intervenção Placebo.

\section{CONCLUSÃO}

Pode-se concluir que a imersão em água fria quando comparada ao placebo, não é uma técnica de recuperação eficaz na redução dos níveis de Creatina Quinase sanguíneo.

\section{REFERÊNCIAS}

Costello JT, Baker PRA, Minett GM, Bieuzen F, Stewart IB, Bleakley C. Whole-body cryother apy (extreme cold air exposure) for preventing and treating muscle soreness after exercise in adults (Protocol). Cochrane Database of Systematic Reviews. 2013; 10: 1-10.

Versey NG, Halson SL, Dawson BT. Water Immersion Recovery for Athletes: Effects on Exercise Performance and Practical Recommendations. Sports Med, 2013; 43(11): 1101-30. https://doi.org/10.1007/s40279-013-0063-8.

Pastre CM, Bastos FN, Netto Júnior J, Vanderlei LCM, Hoshi RA. Métodos de recuperação pósexercício: uma revisão sistemática. Rev Bras Med Esp. 2009; 15(2): 138-144. https://doi.org/10.1590/S1517-86922009000200012.

Bastos, FN. Influência de diferentes tipos de recuperação sobre a modulação autonômica cardíaca, concentração de lactato e proteína C-reativa. Presidente Prudente. Dissertação. [Mestrado em Fisioterapia] - Universidade Estadual Paulista "Júlio de Mesquita Filho", 2009.

Crampton D, Egaña M, Donne B, Warmington SA. Including arm exercise during a cold water immersion recovery better assists restoration of sprint cycling performance. Scandinavian Journal of Medicine \& Science in Sports. 2014; 48(4): 290-98. https://doi.org/10.1111/sms.12169.

Bleakley C, McDonough S, Gardner E, Baxter D, Hopkins Ty, Davison GW. Cold-water immersion (cryotherapy) for preventing and treating muscle soreness after exercise. Sao Paulo Med J. 2012; 130(5): 348. https://doi.org/10.1590/S1516-31802012000500015

Bailey DM, Erith SJ, Griffin PJ, Dowson A, Brewer DS, Gant N, Williams C. Influence of cold-water immersion on indices of muscle damage following prolonged intermittente shuttle running. J Sports Sci. 2007; 25(11): 1163-70. https://doi.org/10.1080/02640410600982659.

Parouty J, Al Haddad H,Quod M, Lepretre PM, Ahmaidi S, Buchheit M. Effect of coldwater immersion on 100-m sprint performance in well-trained swimmers. Eur J Appl Physiol. 2010; 109(3): 483-90. https://doi.org/10.1007/s00421-010-1381-2.

Bleakley CM, Davison GW. What is the biochemical and physiological rationale for using coldwater immersion in sports recovery? A systematic review. Br J Sports Med. 2010; 44(3): 179-87. https://doi.org/10.1136/bjsm.2009.065565. 
Ascensão A, Leite M, Rebelo AN, Magalhäes S, Magalhäes J. Effects of cold water immersion on the recovery of physical performance and muscle damage following a one-off soccer match. J Sports Sci. 2011; 29(3): 217-25.

Ispirlidis I, Fatouros IG, Jamurtas AZ, Nikolaidis MG, Michailidis I, Douroudos I, et al. Time-course of Changes in Inflammatory and Performance Responses Following a Soccer Game. Clin J Sport Med 2008;18(5):423-31.

Yamin C, Amir O, Sagiv M, Attias E, Meckel Y, Eynon N, et al. ACE ID genotype affects blood Creatine Kinase response to eccentric exercise. J Appl Physiol 2007;103(6):2057-61. https://doi.org/10.1152/japplphysiol.00867.2007.

Plebani M. Skeletal muscle biomarkers: not new but still interesting diagnostic tools. Clin Chem Lab Med 2010;48(6):745-6. https://doi.org/10.1515/CCLM.2010.170.

Brancaccio P, Maffulli N, Limongelli FM. Creatine kinase monitoring in sport medicine. Br Med Bull 2007;81-82(1):209-30. https://doi.org/10.1093/bmb/ldm014.

. Jakeman JR, Macrae R, Eston R. A single 10-min bout of cold-water immersion therapy after strenuous plyometric exercise has no beneficial effect on recovery from the symptoms of exerciseinduced muscle damage. Ergonomics 2009;52(4):456-460. https://doi.org/10.1080/00140130802707733.

Skurvydas A, Sipaviciene S, Krutulyte G, Gailiuniene A, Stasiulis A, Mamkus G, Stanislovaitis A. Cooling leg muscles affects dynamics of indirect indicators of skeletal muscle damage. J Back Musculoskelet Rehabil 2006;16: 141-151. https://doi.org/10.3233/BMR-2006-19406.

Stanley J, Buchheit M, Peake JM. The effect of post-exercise hydrotherapy on subsequent exercise performance and heart rate variability. Eur J Appl Physiol. 2012; 112(3): 951-61. https://doi.org/10.1007/s00421-011-2052-7.

Higgins TR, Heazlewood IT, Climstein M. A random control trial of contrast baths and ice baths for recovery during competition in U/20 rugby union. J Strength Cond Res. 2011; 25(4): 1046-51. https://doi.org/10.1519/JSC.0b013e3181cc269f.

McClung M, Collins D. "Because I know it will!" Placebo effects of an ergogenic aid on athletic performance. J Sport Ex Psych. 2007; 29(3): 382-94. https://doi.org/10.1123/jsep.29.3.382

Pinheiro FA, Tróccoli BT, Carvalho CV. Validação do questionário nórdico de sintomas osteomusculares como medida de morbidade. Rev Saúde Pública. 2002; 36(3): 307-12. https://doi.org/10.1590/S0034-89102002000300008.

Stacey DL, Gibala MJ, GinisKAM, Timmons BW. Effects of recovery method after exercise on performance, immune changes and psychological outcomes. Journal of Orthopaedic \&Sports Physical Therapy 2010, 40:10. https://doi.org/10.2519/jospt.2010.3224

Versey NG, Halson SL, Dawson BT. Effect of contrast water therapy duration on recovery of running performance. International Journal of Sports Physlology and Performance 2012, 7, 130140. https://doi.org/10.1123/ijspp.7.2.130. 
Jakeman JR, Macrae R, Eston R. A single 10-min bout of cold-water immersion therapy after strenuous plyometric exercise has no beneficial effect on recovery from the symptoms of exerciseinduced muscle damage. Ergonomics 2009;52(4):456-460.

https://doi.org/10.1080/00140130802707733. 